gravity 1.028. Albumen, none. Sugar, none. $\mathrm{Mi}$ croscopic examination found one granular tube cast from straight uriniferous tubules, also a few epithelia from pelvis of kidney, "some epithelium from bladder and some from vagina. Further than this there was found only the normal inorganic substances, i.e., amorphous urates, urate of ammonia, urate of soda. $573^{\circ}$ Wentworth Ave., Dec. 10, 1886.

\section{THE HEADACHE OF YOUTH AND ITS RELATION TO OCULAR DEFECTS.}

\section{BY CHARLES F. SINCIAIR, M.D.,}

$$
\text { of CHICAGo. }
$$

The subject of my paper is rather the relation of certain common forms of defective vision to headache in youth. The subject in general would take us over the whole field of ophthalmology, and is too vast to treat even superficially in the space allotted to my paper.

The headaches arising from defective vision are so numerous, and show such definite characteristics in connection, not only with the different forms of ametropia, but with its different degrees, that the ophthaimologist might easily furnish a terminology of his own. Instead of the usual classification into sympathetic, neuralgic, anæmic, hyperæmic, etc., another division, based solely upon the condition of the eye, might be made. 'This might be done, too, without regard to any faulty condition of the general system through which these errors of refraction, in many cases, first manifest themselves. And this is true especially in youth. There are headaches with certain definite characteristics which are caused not only by hypermetropia, but also by its different degrees, as there are of myopia and its different degrees. These fixed peculiarities are still more marked in the different forms of astigmatism. The severity of the pain seems in some measure to depend upon the nature of the ametropia, ranging from the dull, heavy ache in and around the eye found in the lighter degrees of hypermetropia and myopia, through the severer forms found in the higher degrees of these refractive errors, and in simple and compound astigmatism, to the intense neuralgic pain of that rare form of defective called mixed astigmatism. This latter pain may be of such severity and of so frequent oc. currence as to destroy the usefulness of the life of the individual.

Such a case as this came under my care in February last, and, although somewhat of a digression, I shall take the liberty of presenting it. This patient was a young woman of 30 years of age, apparently healthy and strong. Her only complaint was about her "terrible head trouble." She had been troubled with her eyes sirce so years of age. When at school, if the sun shone brightly, she could rot read her lessons, and would have to press and squeeze her eyes in order to see at all. About seven years ago her eyes were in such a condition that she could not look at any bright object, or use them for any ordinary work for months at a time. Since she was 15 years of age she has had terrible headaches in the forehead and temples, and in and around the eyes. As she stated, the pain is so intense it almost drives her mad. She can not even ride in a street car for fear the "neuralgia," as she termed it, would start up again.

These severe headaches were accompanied at times with nausea and vomiting, and these she called her "bilious attacks." At other times her "eye headache" came, when the pain seemed to be in and at the back of the eye, and which pressure relieved. At still other times the pain was located at the base of the nose, and these she called her "catarrhal headaches." In all, however, the pain in the eyes was severe. For fifteen years she has had these severe attacks regularly twice a week. They last sometimes a day and a night, and sometimes two days and two nights. She has taken medicine for biliousness and dyspepsia, and every patent medicine she could hear of for her catarrh. She has a large safe at home full of medicine bottles. Her house looks like a drug store. She has exhausted the whole pharmacopaia for lung and liver and stomach and throat troubles, and now, as a last resort, she comes to see if her eyes can be in any measure the cause of her suffering. On examination I found vision very defective, being but $\frac{2}{10}$ in the left eye and $\frac{4}{10}$ in the right. Plus and minus glasses were refused. The ophthalmoscope showed the usual distorted appearance of the disc seen in astigmatic cases, with a moderate degree of myopia in the vertical meridian, and of hypermetropia in the horizontal of both eyes. A lense of r.50 D spherical $\odot+1.50$ cyl. axis vertical in the right eye, and of -2 . D sph. $\odot+1.75 \mathrm{D}$ cyl. axis vertical in the left, improved the vision up to $\frac{7}{T^{*}}$, and apparently has cured the headache.

But it is especially in childhood and youth that these different forms of ametropia manifest them. selves in all the various forms of headache. In a comparatively recent and voluminous work on the "Nature and Cause of Headache," by Wm. Henry Day, of London, a lengthy chapter is devoted to the consideration of the headaches of youth. He deplores the popular methods of education which push a child forward in the acquisition of knowledge without sufficient regard to physical training; "to the vigorous growth of the body and the stability of the frame, until some one link is broken in that mysterious chain of vital force which keeps the whole fabric in order, when the health in too many instances is undermined and life is absolutely shortened."

To one familiar with the intimate relation existing between the headaches of youth and ocular defects, and how, with the school boy and girl, all too frequently the eye is the link which first gives way, it seems strange that the author of such a work should in no instance mention ametropia as a possible cause, not alone of the severe headaches of childhood, but also, through reaction, of deterioration of the general health.

There is, however, one form of ametropia to which, on this occasion, I wish especially to refer, and which is exceedingly common among our school children. It is of slight degree and therefore easily overlooked. Indeed, it may simulate some of the less severe forms 
of ametropia, and although these refractive errors may be but slight, yet they may be the cause of $\mathrm{va}$ rious forms of severe headache and of impairment of the general system. I refer to those cases of myopic and hypermetropic astigmatism of $.5 \circ \mathrm{D}$ and $.75 \mathrm{~L}$. In these cases, for some reason as yet unexplained, the resulting disturbances are much more wide-reaching and serious than in the more common and simpler forms of ametropia.

'The following cases will best illustrate the peculiar characteristics of these forms of abnormal refraction:

Case I.-Mand W., a school girl, 14 years of age, has had severe frontal headache, and occasionally in the occipital region, almost daily for three years. It is usually, however, a severe throbbing pain through the temples and across the forehead. The severity of the pain is so great that she is frequently confined to her bed for days at a time. The headache is sometimes accompanied with nausea. These headaches became finally so frequent and severe that she was obliged to give up all her studies. Nothing during these years of suffering called particular attention to the eyes, but at last she noticed that the eyeballs were sometimes tender to the touch, and that the attacks usually began with slight lancinating pains in and around the eyes.

On examination excellent vision was found, being 9 , with some some few letters of $\frac{1}{10}$ for distance. With minus lenses of .50 and .75 of a dioptric vision improved to such an extent that 10 was read with ease. After, however, the accommodation was paralyzed by the use of homatropin a half a dioptric of hypermetropia was found in the vertical meridian, for which a plus cylinder was prescribed, and no further complaints were made of the severe headache with which this patient had suffered for three years.

Case 2.-Emily R., I 5 years of age, has had headache for a whole year, every day and every night. The pain at first was rather of the nature of a dull, heavy ache in the temples and forehead. This grad. ually increased in severity and extent until at times the whole head seemed numb. 'Two weeks before examination this dull ache turned into a sharp, throbbing, lancinating pain, shooting up through the tem. ples and around the head toward the occiput. There was tenderness on pressure around the orbit, and especially over the supra orbital nerve. The eye-balls were also tender to the touch.

I mention this case for the reason that ordinary plus glasses were first prescribed, these permitting the patient to read $\frac{1}{10}$ with apparent ease. The patient, however, retumed in a few days saying her headaches were as bad as ever. Here also, after the accommodation had been thoroughly paralyzed, a small degree of hypermetropic astigmatism was found which a $\times .50 \mathrm{D}$ cylinder corrected, entirely curing the distressing head symptoms.

The important part these slight irregular forms of ametropia play in the headaches of youth is clearly seen in a case which came under my observation two weeks ago, in which but one eye was slightly astig. matic, the other possessing $\mathbf{1} .50 \mathrm{D}$ of long sight.

Case 3.-Lily L. has been troubled with her eyes for a year and a half. Patient looks delicate and anamic, appetite is poor, has been very much troubled with headache-a dull, continuous ache over the frontal region, with sometimes throbbing and at other times sharp lancinating pains shooting out over the left side of the head from the left eye. During the time this patient attended school these peculiar headaches were of daily occurrence. In vacation they were absent. She is much troubled with con. stantly recurring attacks of vertigo and dizziness. While walking the streets these attacks are liable to come at any moment, when she is obliged to grasp some object to keep from falling.

The greater severity of the symptoms on the left side of the head seemed to find an explanation in the fact that the usual small amount of astigmatism was found in the left eye, the correction of which has apparently put a stop to both the headache and vertigo.

These are but types of, in my opinion, an exceedingly large number of cases where the most distressing symptoms, simulating, in many instances, even organic disease, may be quickly and thoroughly cured by so simple a remedy as a pair of properly adjusted cylindrical lenses. They are interesting to the ophthalmologist, as they may assume in certain cases not only the characteristics of myopia or hypermetropia, but even of normal vision; and they certainly suggest, to the general practitioner the advisability, in every case of severe or persistent headaches in youth, that not only the eye be carefully examined, but that it be examined only when thoroughly under the influence of some mydriatic.

\section{MEDICAL PROGRESS.}

Hypodermatic Injection of Fowien's Solution in Chorea Minor.-To fairly test the value of hypodermatic injections of Fowler's solution of arsenic in the treatment of chorea minor, especially as compared with its internal administration; almost all cases of this disease admitted during the last year to St. Anne's Hospital for Children, in Vienna, service of Prof. Widerhofer, have been subjected to this treatment. For the sake of comparison a few cases were treated by Fowier's solution given internally. The results of the clinical experiment, which have been highly satisfactory, are detailed by DR. FRüHWALD, assistant to Prof. Widerhofer, in the Jahrbuch für Kinderheilkunde. Equal parts of Fowler's solution and distilled water were used; care was taken to have the preparation fresh, and to have it freshly filtered before injecting it. One injection was given a day, alternately into the extremities, the needle being inserted deeply into the tissues and the surface having been well washed with thymol water. Treatment was begun by injecting up to the first division mark of a Pravaz syringe, the dose was increased day by day by one additional part until eight or ten such parts were reached. and then as gradually reduced until the original dose was again attained. In increasing the dose attention was given not only to the age of the patient and to the severity of the disease, but more 